\title{
Estudio de las Actitudes hacia la Estadística en Estudiantes de Psicología
}

\section{Study of Attitudes Toward Statistics in Psychology Students}

\author{
Carles Comas* \\ José Alexandre Martins ${ }^{* *}$ \\ Maria Manuel Nascimento ${ }^{* * *}$ \\ Assumpta Estrada ${ }^{* * * *}$
}

\begin{abstract}
Resumen
La Estadística se ha incorporado en la mayoría de las carreras universitarias y entre ellas en el grado de psicología donde la enseñanza de conceptos estadísticos presenta problemas didácticos específicos debido a que los estudiantes que lo cursan tienen una base matemática muy heterogénea. En este trabajo nos centramos en el estudio de sus actitudes, por su influencia en el proceso de aprendizaje, y las analizamos a través de la Escala de Actitudes hacia la Estadística de Estrada EAEE (ESTRADA, 2002). Los resultados indican actitudes en general moderadas o positivas, con una puntuación promedio global ligeramente superior a la posición teórica de indiferencia. El curso y los estudios previos en esta materia inciden en su actitud. Destacamos que la actitud global hacia la estadística empeora con los años de estudio, aunque las puntuaciones totales más bajas se presentan en los estudiantes que nunca estudiaron estadística, resultados que invitan a la reflexión sobre la manera que se enseñan en los diferentes niveles educativos.
\end{abstract}

Palabras clave: Actitudes. Escalas. Estadística. Estudiantes de Psicología.

\begin{abstract}
Statistics has been incorporated in most university courses and among them in the degree of Psychology, where the teaching of statistical concepts presents specific learning problems, because students enrolled have a very heterogeneous mathematical basis. In this work, we focus on the study of those students' attitudes for their influence on the learning process and we analyze it through the Scale of Attitudes toward Statistics of Estrada,
\end{abstract}

\footnotetext{
* Doctor en Estadística por la University of Strathclyde (Reino Unido). Profesor Agregado. Departamento de Matemática, Facultad de Educación, Psicologia y Trabajo Social de la Universitat de Lleida (UdL), Lleida, España. Dirección postal: Facultad de Educación, Psicologia y Trabajo Social, Av. Estudi General, 4, 25002, Lleida, España. E-mail: carles.comas@matematica.udl.cat.

** Doutor em Didática das Ciências e Tecnologias, Especialização em Didática das Matemáticas pela Universidade de Trás-os-Montes e Alto Douro (UTAD). Investigador integrado na Unidade de Investigação para o Desenvolvimento do Interior do Instituto Politécnico da Guarda (UDI/IPG), Guarda, Portugal. Dirección postal: Escola Superior de Turismo e Hotelaria, Rua Dr. José António Fernandes Camelo, Arrifana, 6270-372, Seia, Portugal. E-mail: jasvm@ipg.pt.

Doutora em Matemática pela Universidade de Trás-os-Montes e Alto Douro (UTAD). Professora do Departamento de Matemática de Universidade de Trás-os-Montes e Alto Douro e membro do Centro de Investigação "Didática e Tecnologia na Formação de Formadores" (CIDTFF). Dirección postal: UTAD, Quinta de Prados, 5000-801, Vila Real, Portugal. E-mail: mmsn@utad.pt.

**** Doctora en Didáctica de las Matemáticas por la Universidad Autónoma de Barcelona (UAB). Profesora Titular de Universidad del Departamento de Matemática, Facultad de Educación, Psicologia y Trabajo Social, Universitat de Lleida (UdL), Lleida, España. Dirección postal: Facultad de Educación, Psicologia y Trabajo Social, Av. Estudi General, 4, 25002, Lleida, España. E-mail: aestrada@matematica.udl.cat.
} 
Spanish acronym EAEE (ESTRADA, 2002). The results indicate moderate or positive attitudes in general with an overall average score slightly higher than the theoretical position of indifference. The course and the previous studies in this area affect their attitude. We emphasize that the overall attitude towards statistics is worsened by the increase of the number years of studies, although the total lowest scores are presented in students who never studied statistics, results that invite us to reflect on how statistics has been taught through the different educational levels.

Keywords: Attitudes. Scales. Statistics. Psychology students.

\section{Introducción}

La Estadística se ha incorporado, en forma generalizada, al currículum de la mayoría de estudios universitarios como fruto del importante papel que desempeña en la formación científica y técnica de profesionales de muy variado perfil. Su estudio ayuda al desarrollo personal, fomentando un razonamiento crítico, basado en la valoración de la evidencia objetiva; así, hemos de ser capaces de usar los datos para controlar nuestros juicios e interpretar los de los demás. Es importante adquirir un sentido de los métodos y razonamientos que permiten trasformar estos datos para resolver problemas de decisión y efectuar predicciones (OTTAVIANI, 1999).

En cuanto a lo que a los estudiantes de Psicología se refiere, según Vera y Díaz (2013) la enseñanza de los conceptos estadísticos plantea especiales problemas didácticos, al no poseer una base matemática tan amplia como estudiantes de otras carreras científicas. Pero como señala Batanero (1999), lo verdaderamente importante no son solamente los contenidos específicos, sino tratar de desarrollar en nuestros alumnos una actitud favorable, unas formas de razonamiento y un interés por completar posteriormente su aprendizaje.

Las actitudes son parte integrante de todas las materias de aprendizaje y ocupan un lugar central en el acto educativo, guiando el proceso perceptivo y cognitivo que comporta el aprendizaje de cualquier contenido educativo.

El trabajo que aquí presentamos se centra en el estudio de las actitudes hacia la Estadística de estudiantes de Psicología de una universidad pública española. Analizamos las respuestas a los ítems de la Escala de Actitudes hacia la Estadística de Estrada EAEE (ESTRADA, 2002) con el objetivo de analizar la actitud hacia la Estadística de estos estudiantes, compararla con las de otros colectivos afines, así como su relación con variables personales y escolares.

\section{Las actitudes y sus componentes}


Los trabajos de McLeod $(1988,1989,1992,1994)$ son el principio de una corriente en educación matemática que reconoce la importancia de las cuestiones afectivas en el aprendizaje. El autor indica que las actitudes tienen una definición difícil, debido a que no son directamente observables, sino que se trata de construcciones teóricas que se infieren de los comportamientos de los sujetos.

Posteriormente, encontramos diversas definiciones de las actitudes. Gal y Garfield (1997, p.40) las conciben como "una suma de emociones y sentimientos que se experimentan durante el período de aprendizaje de la materia objeto de estudio". Gómez (2000, p.23) considera la actitud como: "una predisposición evaluativa (es decir positiva o negativa) que determina las intenciones personales e influye en el comportamiento". Por otro lado, ya más recientemente Phillip (2007) las describe como formas de actuar, sentir o pensar que muestran la disposición u opinión de una persona.

Las actitudes hacia un determinado tema, por ejemplo, la estadística, suelen ser estables, se pueden graduar según su intensidad, ser positivas o negativas (agrado o desagrado, gusto o disgusto por el tema) y, en ocasiones, expresan sentimientos vinculados a elementos que no son estrictamente parte de la materia (como el profesor o tipo de actividad). Surgen en edades muy tempranas, y aunque tienden a ser favorables en un principio, pueden evolucionar en forma negativa con el paso del tiempo. Diversos estudios indican que su origen proviene de:

- El conocimiento del tema adquirido a partir de la vida cotidiana fuera del aula; en el caso de la estadística, podría ser a partir de la prensa o en los medios de comunicación (GAL; GINSBURG, 1994).

- Las experiencias previas de aprendizaje en contexto escolar; por ejemplo, si la enseñanza recibida fue rutinaria, o no se mostraron aplicaciones reales adecuadas.

- Su vinculación con las Matemáticas. Al considerar que la Estadística es parte de las Matemáticas, se transfieren las actitudes de una materia a otra.

Las primeras investigaciones sobre actitudes las consideraban como un constructo unidimensional, pero progresivamente se han utilizado modelos multidimensionales de las actitudes. Así, Wise (1985) usó solamente dos componentes diferenciados: (a) las actitudes hacia el curso de Estadística básica que están realizando los alumnos (componente curso), y (b) las actitudes de los alumnos hacia el uso de la Estadística en su campo de estudio (componente campo). 
Auzmendi (1992), Gil Flores (1999) y Gómez (2000) diferencian tres factores básicos en las actitudes (que denominaremos componentes pedagógicos):

- Componente cognitivo: se refiere a las concepciones y creencias, acerca del objeto actitudinal, en este caso, la Estadística.

- Componente afectivo o emocional: recogería las emociones y sentimientos que despierta la Estadística, y por ello son más subjetivas; por ejemplo, sentimientos de rechazo o de interés.

- Componente conductual o tendencial: representan la tendencia a la acción o intención de una manera determinada; por ejemplo, cómo y cuándo se usaría la estadística.

En nuestro trabajo, también partimos de un concepto pluridimensional de las actitudes de los profesores hacia la Estadística, contemplando los componentes pedagógicos descritos anteriormente. Además se consideran otros componentes que llamamos antropológicos:

- Componente social: actitudes relacionadas con la percepción y valoración del papel de la Estadística en el ámbito sociocultural de cualquier ciudadano;

- Componente educativo: analizamos en este componente el interés hacia la Estadística y su aprendizaje, la visión de su utilidad para el alumno, su opinión sobre si debiera ser incluida en el currículo y la dificultad percibida;

- Componente instrumental: se recoge, aquí, la utilidad hacia otras materias, como forma de razonamiento y como componente cultural.

Nuestro propósito al introducir estos nuevos componentes fue, además de contemplar todas aquellas cuestiones referentes a la utilidad, formación y multidisciplinariedad de la estadística, recoger también todas aquéllas que la relacionan directamente con el trasfondo social y cultural.

El análisis de las actitudes hacia la Estadística, en España y a nivel internacional, tiene ya una cierta tradición, sobre todo en las dos últimas décadas. Un análisis detallado de estas investigaciones previas aparece en Estrada (2002, 2007, 2009), y es complementado por Carmona (2004) con el estudio de las características psicométricas de una serie de escalas de medición de actitudes y de las evidencias de relación de las actitudes con diferentes variables externas.

En general, estas investigaciones se han orientado fundamentalmente hacia la construcción de escalas de medición de actitudes, entre las que destacamos el SAS de Roberts y Bilderback (1980), el ATS de Wise (1985) y el SATS de Schau et al., (1995), por ser las más utilizadas. Otros trabajos analizan la influencia de diversas variables tales como el género 
(ANASTASIADOU, 2005), el rendimiento académico (NASSER, 2004), la experiencia formativa en Matemáticas y Estadística (AUZMENDI, 1992; ELMORE; VASU, 1980, 1986), el área de estudios (GIL FLORES, 1999).

Dentro de estos antecedentes, destacan los estudios referidos a los profesores, en formación o en ejercicio. Así, los trabajos de Onwuegbuzie, (1998, 2003), Watson (2001), Nasser (2004), Aparicio y Bazán (2006a, 2006b, 2008), Lancaster (2008) y, en España, los de Estrada y colaboradores (ESTRADA, 2002, 2007; ESTRADA, BATANERO; FORTUNY, 2003, 2004; ESTRADA; BATANERO, 2008; MARTINS; NASCIMENTO; ESTRADA, 2009; ESTRADA; BATANERO; LANCASTER, 2011 insertar en las REFS) dedican su atención a este colectivo, estudiando sus actitudes juntamente con otras variables. En el ámbito de los estudiantes de psicología, colectivo afín al de los profesores, son escasos los trabajos encontrados en la revisión bibliográfica realizada, destacamos, por su especificidad, los de Morales (1988) y Cuesta, Rifá y Herrero (2001).

\section{Metodología}

El instrumento de medición de actitudes utilizado en este trabajo es la escala de actitudes hacia la Estadística de Estrada (2002), el EAEE, aplicada en diferentes países a estudiantes universitarios de diversas titulaciones aunque no de piscología. Dicha escala se construyó combinando tres instrumentos de medición de actitudes: Escala SAS (Statistics Attitudes Survey, de ROBERTS; BILDERBACK, 1980); Escala ATS (Attitude Towards Statistics, de WISE,1985) y la de Auzmendi (1992).

A partir de las tres escalas citadas, se consiguió un listado de 36 enunciados, que se sometieron a un panel de jueces, expertos con diferentes perfiles profesionales y que emiten su opinión respecto a la adecuación y univocidad de las sentencias. Después de la validación, la escala definitiva se reduce a 25 ítems, 14 afirmativos frente a 11 negativos, y cuya distribución según los componentes evaluados por cada ítem es la que aparece en la Tabla 1. Los ítems constan de un enunciado y una escala tipo Likert de 5 puntos, que valora las respuestas desde muy en desacuerdo (1 punto) hasta muy de acuerdo. Se fue intentando incluir, tanto ítems redactados en forma afirmativa (la Estadística ayuda a entender el mundo de hoy), como otros en forma negativa (en la escuela no se tendría que enseñar Estadística). Y todo ello para evitar el problema de la aquiescencia (MORALES, 1988), por el que algunos sujetos tienden a responder con la forma de acuerdo sea cual sea el contenido del ítem.

Tabla 1- Componentes de las actitudes evaluadas en la escala EAEE (2002) 


\begin{tabular}{lccc}
\hline & \multicolumn{3}{c}{ Componentes antropológicos } \\
\hline Componentes pedagógicos & Social & Educativa & Instrumental \\
Afectivo & $1,11,25$ & $7,12,23$ & $10,13,16,20$ \\
Cognitivo & $2,19,21$ & $4,6,17$ & 3,24 \\
Comportamental & 9,18 & $8,15,22$ & 5,14 \\
\hline
\end{tabular}

Fuente: Estrada (2002)

La recogida de datos se realizó en el segundo semestre del curso 2013-2014, a un total de 142 sujetos en los tres primeros cursos de la titulación de Psicología de una universidad española, y se obtuvieron 108 cuestionarios completos.

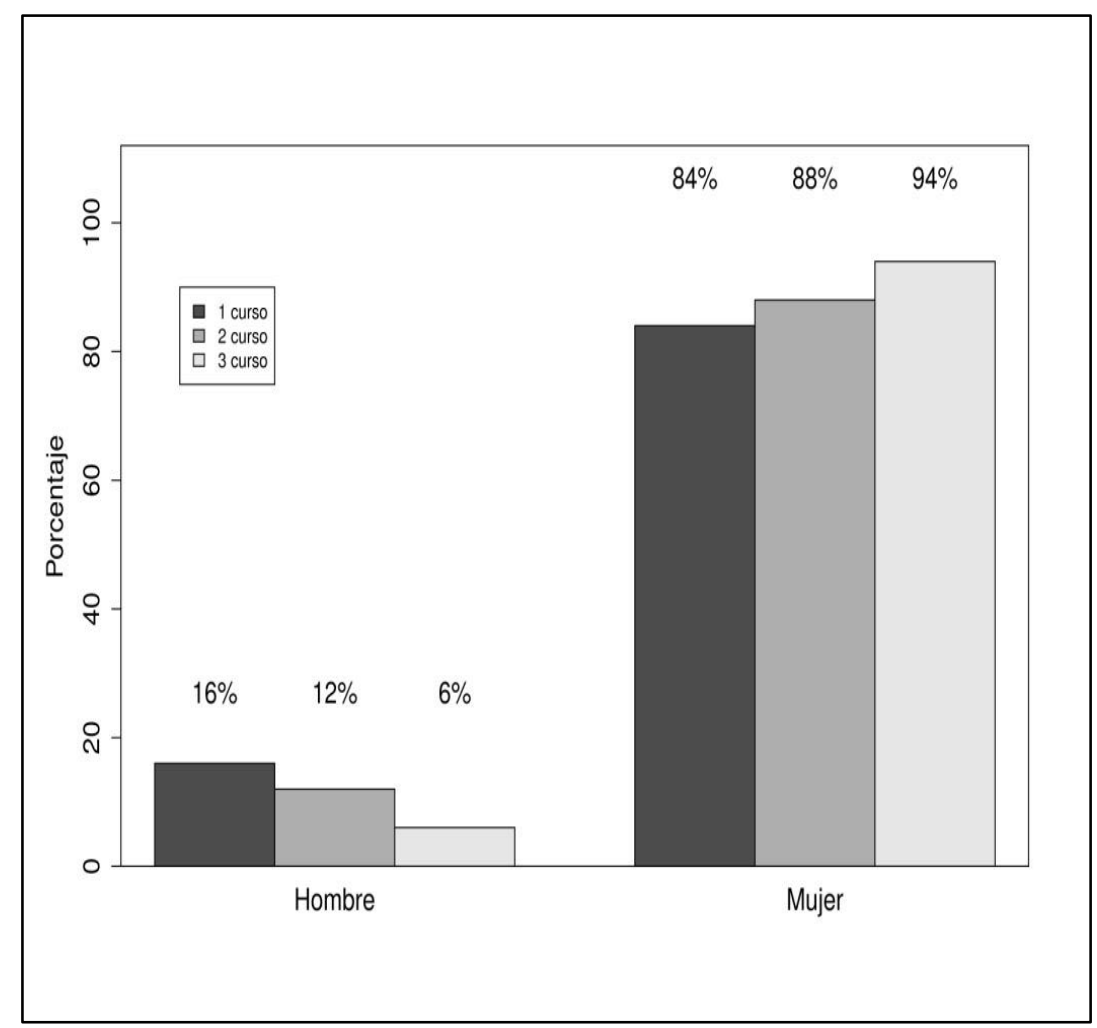

Figura 1 - Distribución de la muestra por género y curso Fuente: Construcción propia

En la muestra recogida hay una fuerte incidencia del colectivo de mujeres, situación habitual en este tipo de carreras, de manera que en $3^{\circ}$ curso llegan a alcanzar un $94 \%$ tal como se puede observar en la Figura 1, donde se presenta la distribución del género por cursos.

Con respecto a la formación estadística de estos alumnos según el número de etapas, recordemos que la variable estudios previos toma los valores categóricos $0,1,2$ y 3 , donde el cero significa que antes de la universidad no la estudió. 


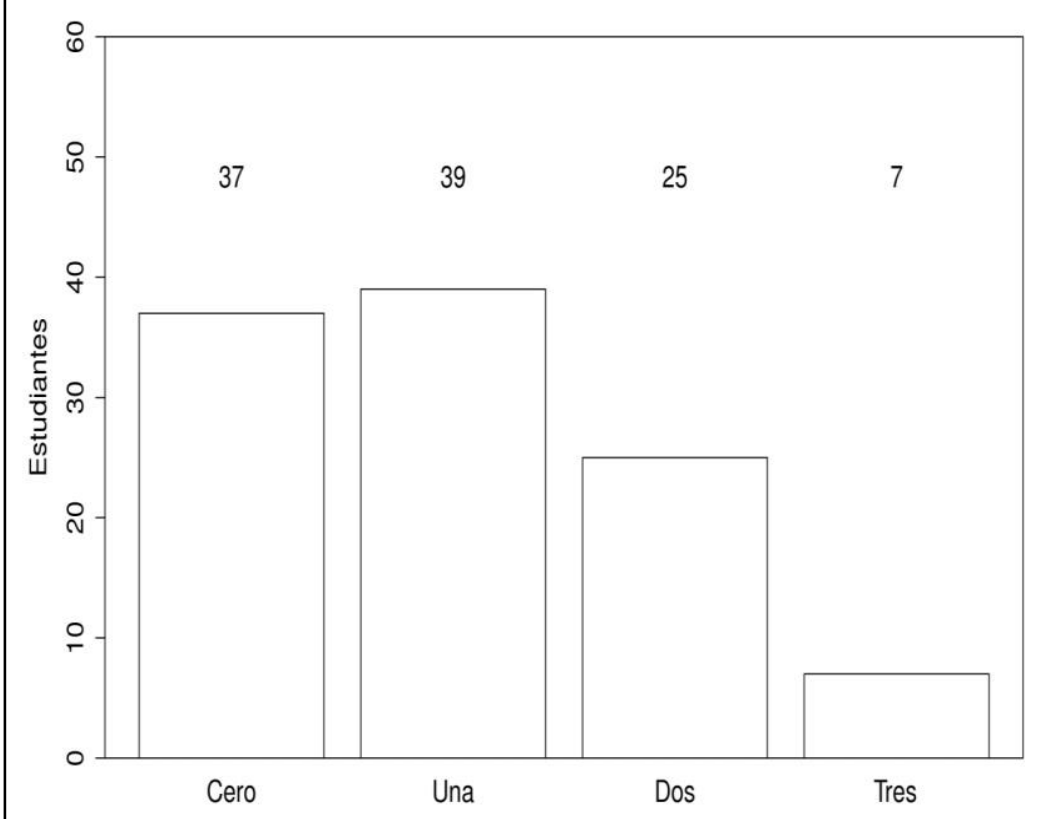

Figura 2 - Distribución de la muestra por número de etapas de estudios previos donde se cursó estadística Fuente: Construcción propia

En la Figura 2 nos parece interesante resaltar como a pesar de que oficialmente la Estadística está presente en los diferentes niveles educativos (Primaria/ESO/ Bachillerato o FP), sólo 7 futuros psicólogos la estudiaron en los tres niveles, un 33\% (37) llegan a la universidad sin haberla tratado en toda su vida académica y entre los que la estudiaron, sólo 12 de los alumnos encuestados la estudia en Primaria (Figura 3).

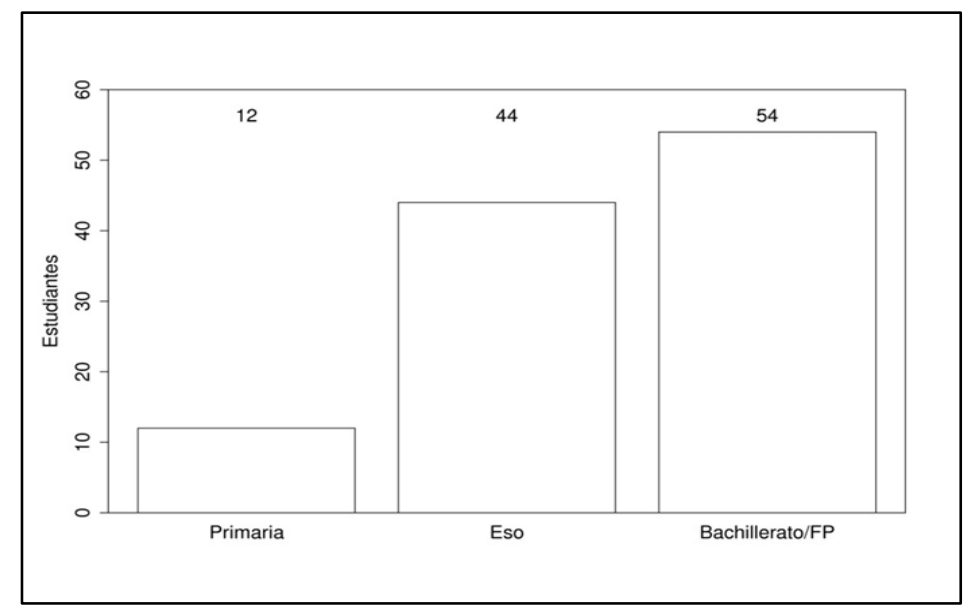

Figura 3 - Distribución de los estudiantes con estudios previos según etapa Fuente: Construcción propia

\section{Resultados y discusión}


Una vez presentados los datos de las características de la muestra participante, analizamos los resultados referentes a cada uno de los veinticinco ítems, tal como fueron presentados a los sujetos de la muestra (Tabla 2).

Tabla 2 - Frecuencias de respuestas, media y desviación típica en cada ítem

\begin{tabular}{|c|c|c|c|c|c|c|c|}
\hline Enunciado del ítem & 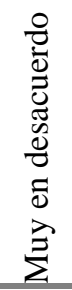 & 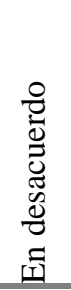 & 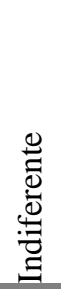 & 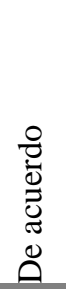 & 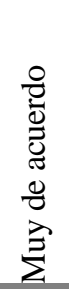 & 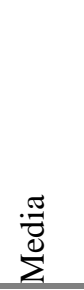 & 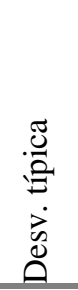 \\
\hline $\begin{array}{l}\text { 1. Me molesta la información estadística que aparece en algunos } \\
\text { programas de T.V. }\end{array}$ & & 12 & 50 & 30 & 15 & 3,43 & 0,90 \\
\hline 2. La Estadística ayuda a entender el mundo de hoy. & 1 & 12 & 16 & 69 & 10 & 3,69 & 0,83 \\
\hline 3. A través de la Estadística se puede manipular la realidad. & 25 & 50 & 18 & 12 & 3 & 2,24 & 1,02 \\
\hline 4. Es fundamental en la formación básica del futuro ciudadano. & 5 & 18 & 48 & 36 & 1 & 3,09 & 0,85 \\
\hline 5. Uso la Estadística para resolver problemas de la vida cotidiana. & 20 & 38 & 22 & 25 & 3 & 2,56 & 1,12 \\
\hline 6. En la escuela no se tendría que enseñar Estadística. & 1 & 2 & 25 & 52 & 28 & 3,96 & 0,81 \\
\hline 7. Me divierto en las clases en que se explica Estadística. & 23 & 28 & 35 & 18 & 4 & 2,56 & 1,11 \\
\hline 8. Los problemas de Estadística me resultan fáciles. & 14 & 27 & 38 & 28 & 1 & 2,77 & 1,01 \\
\hline $\begin{array}{l}\text { 9. No entiendo las informaciones estadísticas que aparecen en la } \\
\text { prensa. }\end{array}$ & & 8 & 20 & 66 & 13 & 3,76 & 0,80 \\
\hline $\begin{array}{l}\text { 10. Me gusta la Estadística porque me ayuda a comprender más } \\
\text { profundamente la complejidad de ciertos temas. }\end{array}$ & & 20 & 45 & 36 & 4 & 3,17 & 0,87 \\
\hline 11. Me siento intimidado ante datos estadísticos. & 1 & 12 & 30 & 41 & 24 & 3,69 & 0,97 \\
\hline 12. Encuentro interesante el mundo de la Estadística. & 12 & 24 & 28 & 36 & 8 & 3,04 & 1,14 \\
\hline $\begin{array}{l}\text { 13. Me gustan los trabajos serios en que aparecen estudios } \\
\text { estadísticos. }\end{array}$ & & 14 & 36 & 41 & 10 & 3,31 & 1,03 \\
\hline 14. Utilizo poco la Estadística fuera de la escuela. & 32 & 38 & 16 & 18 & 4 & 2,30 & 1,17 \\
\hline 15. En clase de Estadística nunca entiendo de qué están hablando. & 4 & 9 & 29 & 44 & 22 & 3,66 & 1,02 \\
\hline $\begin{array}{l}\text { 16. Me apasiona la Estadística porque ayuda a ver los problemas } \\
\text { objetivamente. }\end{array}$ & & 26 & 45 & 25 & 3 & 2,88 & 0,95 \\
\hline 17. La Estadística es fácil. & 17 & 38 & 34 & 17 & 2 & 2,53 & 1,00 \\
\hline $\begin{array}{l}\text { 18. Me entero más del resultado de las elecciones cuando aparece } \\
\text { representaciones gráficas. }\end{array}$ & & 4 & 15 & 54 & 34 & 4,07 & 0,83 \\
\hline 19. La Estadística sólo sirve a la gente de ciencias. & 2 & 1 & 17 & 56 & 32 & 4,06 & 0,81 \\
\hline 20. Me gusta hacer problemas cuando uso la Estadística. & 12 & 30 & 40 & 23 & 3 & 2,77 & 1,00 \\
\hline 21. La Estadística no sirve para nada. & 0 & 4 & 12 & 42 & 50 & 4,28 & 0,81 \\
\hline $\begin{array}{l}\text { 22. A menudo explico a mis compañeros problemas de Estadística que } \\
\text { no han entendido. }\end{array}$ & & 32 & 23 & 25 & 7 & 2,68 & 1,21 \\
\hline 23. Si pudiera eliminar alguna materia, sería la Estadística. & 11 & 13 & 27 & 28 & 29 & 3,47 & 1,29 \\
\hline 24. La Estadística ayuda a tomar decisiones más documentadas. & 2 & 8 & 29 & 58 & 11 & 3,63 & 0,84 \\
\hline 25. Evito las informaciones estadísticas cuando las leo. & 2 & 14 & 32 & 49 & 11 & 3,49 & 0,91 \\
\hline
\end{tabular}

Fuente: Elaborada por los autores

En la Tabla 2 hacemos constar el número de casos de cada una de las categorías $(1=$ muy en desacuerdo, 2 = en desacuerdo, 3 = indiferente, $4=$ de acuerdo, $5=$ muy de acuerdo) para el total de la muestra. Presentamos, también, las medias y desviaciones típicas de las 
puntuaciones obtenidas con el criterio anterior. Hacemos notar que los ítems 1, 3, 6, 9, 11, $14,15,19,21,23$ y 25 , tienen un enunciado negativo y, por ello, en el momento del vaciado de los cuestionarios se invirtió la puntuación $(1=$ muy de acuerdo, $2=$ de acuerdo, $3=$ indiferente, $4=$ en desacuerdo, $5=$ muy en desacuerdo) para poder interpretar sus resultados correctamente.

Esta decisión se toma porque, en el cálculo de la puntuación total, es necesario que todos los ítems tengan la misma dirección y, sobre todo, para poder tener una escala homogénea de comparación de todos los ítems, en la que una media más (o menos) alta indique siempre una actitud más (o menos) positiva, independientemente de si el ítem se redacta con enunciado positivo o negativo. De esta manera, la puntuación total (suma de las puntuaciones de los 25 ítems) representará la actitud de cada encuestado respecto a la Estadística y, ésta será tanto más favorable cuanto más elevada sea esta puntuación.

Entre los ítems mejor valorados, con 4,28 puntos, destacamos el ítem 21 (la Estadística no sirve para nada). Corresponde a un componente cognitivo y social que pone de manifiesto el valor que el colectivo otorga a la materia, al ser un ítem de formulación negativa significa que los estudiantes no están de acuerdo con la afirmación, y la consideran de utilidad, resultado en concordancia con los de Tanur (1992), donde se presenta la Estadística como una potente herramienta de trabajo multidisciplinar, utilizadas en muy diferentes ámbitos.

Además si lo interpretamos conjuntamente con el siguiente mejor valorado $(4,07)$, el ítem 18 (Me entero más del resultado de las elecciones cuando aparecen representaciones gráficas), se refuerza la importancia que se otorga a la presencia de la Estadística en la vida cotidiana, otro de los pilares básicos que justifican su inclusión en la enseñanza obligatoria, como formación básica de todos los ciudadanos (GAL, 2002).

Con una puntuación ligeramente inferior (4,06), el ítem 19 (La Estadística sólo sirve para la gente de ciencias) corresponde una vez más a aspectos cognitivos inducidos por un componente antropológico social, y significa que los futuros psicólogos aprecian la utilidad de la estadística en titulaciones que no son específicamente de ciencias. Estos resultados coinciden con los de Nascimento, Martins y Estrada (2012), realizados con otros colectivos de estudiantes universitarios en los que también aparecía como uno de los ítems mejor valorados. Finalmente, nos parece interesante destacar que todos los items del componente social presentan puntuaciones positivas en todos sus componentes pedagógicos. Estos resultados refuerzan la idea de que es positiva la percepción y valoración del papel de la Estadística en el ámbito sociocultural de los ciudadanos. 
Entre los ítems que no alcanzan una puntuación 3 (actitud neutra), el peor de todos es el ítem 3 (A través de la Estadística se puede manipular la realidad), con 2,24 y sentencia negativa lo que sugiere desconfianza hacia los datos estadísticos, en coincidencia con numerosos autores, que señalan que la Estadística cuenta, a veces, con mala imagen, debido al abuso que de ella se hace en publicidad o en política.

A continuación, el ítem 14 (Utilizo poco la estadística fuera de la escuela) con 2,30 que junto con el 5 (Uso la Estadística para resolver problemas de la vida cotidiana) con 2,56 corresponden a un componente antropológico instrumental y revela una actitud negativa en términos de acción por medio del uso de la estadística (componente comportamental asociado), estos resultados son aun inferiores $(2,55)$ y $(2,89)$ respectivamente, a los de Nascimento, Martins y Estrada (2012).

Las peores valoraciones del componente afectivo son el ítem 7 (Me divierto en las clases en que se explica Estadística) con 2,56, combinado con el educativo y el 16 con 2,88 (Me apasiona la Estadística porque ayuda a ver los problemas objetivamente) y 20 con 2,77 (Me gusta hacer problemas cuando uso la Estadística) con el componente instrumental lo que refleja la influencia de los afectos en el uso de la estadística en el aula para resolver problemas. Con una puntuación de 2,53 encontramos el ítem 17 (La Estadística es fácil) que indica, según trabajos previos, no una falta de la propia disciplina, sino de la manera en que se enseña.

Habría que seguir las recomendaciones de Moore (1987), quien sugiere, por un lado, cambiar los contenidos, y enseñar una Estadística basada en los datos, con menor énfasis en la probabilidad que resulta más difícil a los alumnos; y por el otro, cambiar la metodología, introduciendo la tecnología y el trabajo con proyectos. Finalmente, el ítem 8 con 2,77 (Los problemas de Estadística me resultan fáciles) y el 22 (A menudo explico a mis compañeros problemas de Estadística que no han entendido) con 2,68 referente al componente educativo y comportamental indican que los estudiantes, al no resultarles fáciles resolver problemas, no se ven capaces de explicarles a sus compañeros, generalmente son los que aplazan para otro momento las tareas o esperan que otros les ayuden o las hagan si es un trabajo de grupo.

Una vez analizados los resultados por ítem, se hizo un estudio de fiabilidad por medio del programa, obteniendo un valor del coeficiente de consistencia interna, $\alpha$ de Cronbach, de 0,878 a nuestro entender suficiente para el estudio, teniendo en cuenta el tamaño restringido de la muestra. 


\subsection{Resultados globales}

En la Figura 4 presentamos el histograma de frecuencias de la puntuación total en la escala de actitudes hacia la Estadística para el total de la muestra.

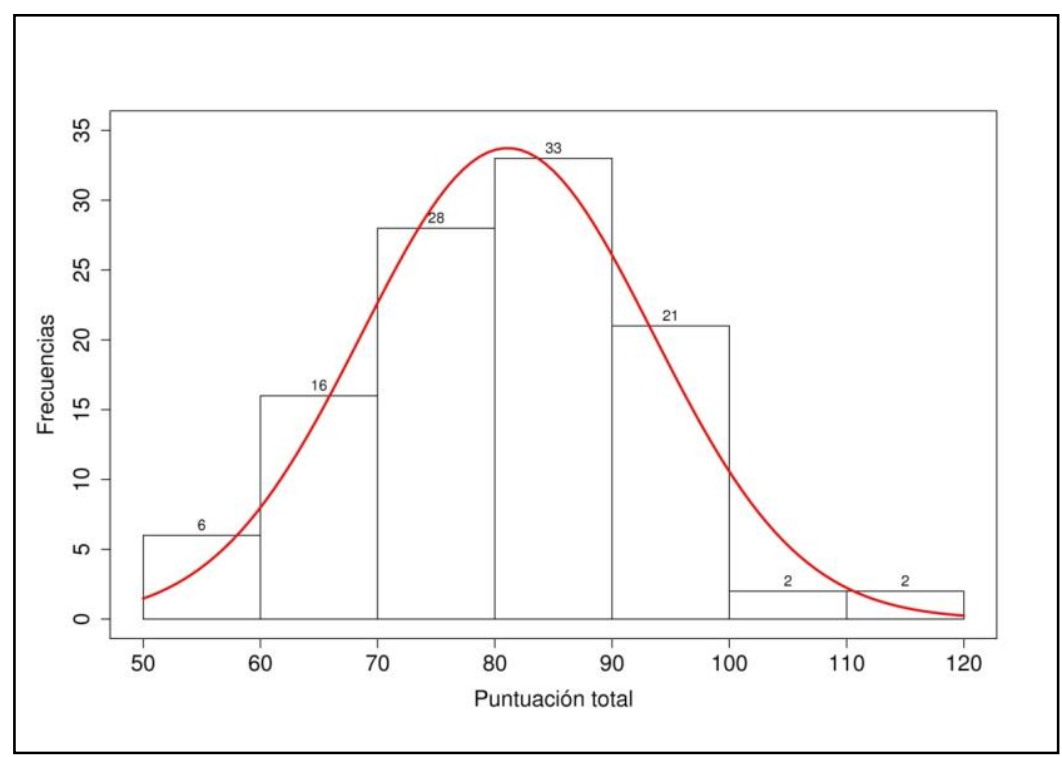

Figura 4- Distribución de frecuencias de la puntuación total Fuente: Construcción propia

A la vista de los resultados obtenidos, y teniendo en cuenta que la puntuación correspondiente a la posición de indiferencia es 75, podemos afirmar que la actitud de los encuestados respecto a la Estadística es positiva, ya que solamente 28 individuos obtienen puntuaciones inferiores a este valor y la gran mayoría se concentra en una actitud positiva alrededor de 85 (moda). El valor de la media 81.08 es moderadamente positivo y podemos observar en el histograma la forma aproximadamente normal de la distribución de la puntuación total que nos autorizará al uso de los programas de análisis de varianza. Asimismo, hemos obtenido un valor tipificado del coeficiente de asimetría (0.058) y curtosis $(0,262)$ dentro de los límites [-2,2] admitidos dentro de los límites de normalidad.

\subsection{Relación entre las variables curso y género, y la actitud hacia la Estadística}

En la Tabla 3 presentamos los resultados del análisis de varianza factorial con dos factores intersujetos: género (con dos niveles) y curso (con tres niveles).

Tabla 3 - Análisis de varianza de la puntuación total en función de género y curso

\begin{tabular}{llcccc}
\hline Fuente & $\begin{array}{l}\text { Suma } \\
\text { de cuadrados }\end{array}$ & gl & Media cuadrática & F & Sig. \\
\hline Curso & 865,8 & 1 & 865,8 & 5,97 & 0,016 \\
Género & 541,5 & 1 & 541,5 & 3,73 & 0,055
\end{tabular}




$\begin{array}{lccccc}\text { Curso*Género } & 25,8 & 1 & 25,76 & 0,17 & 0,674 \\ \text { Error } & 15061,2 & 104 & 144,82 & & \end{array}$

Fuente: Elaborada por los autores

A la vista de los resultados obtenidos, observamos que sólo el curso tiene un efecto estadísticamente significativo sobre la puntuación total en la escala de actitudes, no así el género ni la interacción entre ambos, lo que confirma estudios previos realizados en colectivos afines.

En la tabla 4 presentamos las medias, desviaciones típicas e intervalos de confianza totales, según curso y género, que nos ayudarán a completar el estudio.

Tabla 4 - Medias, desviaciones típicas e intervalos de confianza para las variables género y curso

\begin{tabular}{cccccc}
\hline & & Media & Error. típ. & \multicolumn{2}{c}{ Intervalo de confianza al 95\%. } \\
\hline \multirow{2}{*}{ TOTAL } & & & Límite inferior & Límite superior \\
GÉNERO & & 81,08 & 0,33 & 80.43 & 81,73 \\
& Hombre & 87,41 & 0,79 & 85,86 & 88,96 \\
\multirow{3}{*}{ CURSO } & Mujer & 80,29 & 0,36 & 79,58 & 81.00 \\
& Primero & 87,31 & 0,57 & 86,19 & 88,43 \\
& Segundo & 77,78 & 0,52 & 76,76 & 78,80 \\
& Tercero & 79,29 & 0,61 & 78,09 & 80,49 \\
\hline
\end{tabular}

Fuente: Elaborada por los autores

De acuerdo con los resultados del análisis de varianza, hay una diferencia de casi 7 puntos en puntuaciones medias entre hombres y mujeres, lo que contradice los resultados de otras investigaciones anteriores de Estrada, Batanero y Fortuny (2004) y confirman los de Cazorla et al. (1999), donde también indican que la actitud hacia la Estadística es peor en el caso de las mujeres.

En el caso de la variable curso llama poderosamente la atención como la actitud más positiva es la de los alumnos de primero y disminuye, curiosamente, cuando la formación aumenta lo que corrobora los estudios de Moore (1987) citados anteriormente y sus recomendaciones metodológicas sobre la manera en que se enseña esta materia.

\subsection{Influencia de la variable estudios previos}

En la Tabla 5 mostramos los resultados del análisis factorial de la varianza respecto a estudios previos con 4 niveles, que toma los valores categóricos $0,1,2$ y 3 correspondientes a las diferentes etapas en las que era posible estudiar Estadística. 
Tanto el curso como los estudios previos, tienen un efecto estadísticamente significativo sobre la puntuación media en la escala de actitudes, pero no así la interacción entre ambos. Estos resultados, que pueden resultar extraños, tienen sentido pues la formación recibida por estos alumnos es homogénea y no ha estado sometida a cambios de planes de estudio que afecten a esta materia, en general son alumnos que realizaron la LOGSE y, tal como señalan Estrada, Bazán y Aparicio (2012), es la gran ausente dentro de la enseñanza obligatoria en España.

Tabla 5 - Análisis de varianza en función de estudios previos y el curso

\begin{tabular}{lccccc}
\hline Fuente & $\begin{array}{l}\text { Suma } \\
\text { de cuadrados }\end{array}$ & gl & Media cuadrática & F & Sig. \\
\hline Estudios previos & 853,3 & 1 & 853,2 & 6,082 & 0,0152 \\
Curso & 1026,1 & 1 & 1026,1 & 7,314 & 0,0079 \\
Estudios previos*Curso & 25,0 & 1 & 25,0 & 0,178 & 0,6734 \\
Error & 14589.7 & 104 & 142.11 & & \\
& & & & &
\end{tabular}

Fuente: Elaborada por los autores

Puesto que la variable estudios previos (4 niveles según número de etapas) es cuantitativa, para estudiar su efecto sobre la puntuación total hemos calculado el coeficiente de correlación de Pearson, su resultado es 0.85 con un valor de $\mathrm{p}=0.15$, pero dado el tamaño reducido de la muestra, se espera la no significación del test. Finalmente, en la Tabla 6 presentamos las medias, desviaciones típicas e intervalos de confianza para la variable estudios previos, cuyos resultados indican que la actitud es claramente más positiva en aquellos alumnos que han cursado Estadística en los tres niveles establecidos en el curriculum.

Tabla 6 - Medias, desviaciones típicas e intervalos de confianza para la variable Estudios Previos

\begin{tabular}{ccccc}
\hline & Media & Error. típ. & \multicolumn{2}{c}{ Intervalo de confianza al 95\%. } \\
\hline & & \multicolumn{4}{c}{ Límite inferior } & Límite superior \\
$\begin{array}{c}\text { Total } \\
\text { Estudios previos }\end{array}$ & 81,08 & 0,33 & 80.43 & 81,73 \\
0 & 79.10 & 0,60 & 77.91 & 80.29 \\
1 & 80.07 & 0,55 & 78.97 & 81,17 \\
2 & 81.44 & 0,67 & 80.11 & 82.77 \\
3 & 95.85 & 1,04 & 93.80 & 97.90 \\
\hline \multicolumn{4}{l}{}
\end{tabular}

\section{Conclusiones}

En primer lugar, queremos indicar que las actitudes en general fueron moderadas o positivas, con una puntuación promedio global ligeramente superior a la posición teórica de indiferencia. Mientras que las investigaciones de Auzmendi (1992), Wilensky (1997) indican 
que los alumnos muestran actitudes negativas hacia la estadística, nuestro estudio con futuros psicólogos se aparta de esta tendencia y está más en línea, aunque inferiores con los resultados de Cuesta, Rifá y Herrero (2001), con el mismo colectivo o más recientemente los de Estrada, Bazán y Aparicio (2010), o Nascimento, Martins y Estrada (2012).

A la vista de los resultados obtenidos, y de acuerdo con los resultados del análisis de varianza, las variables curso y estudios previos interaccionan entre si, y son estadísticamente significativas.

En el caso del género, las diferencias existentes indican que la actitud hacia la estadística es peor en el caso de las mujeres, en contradicción con investigaciones previas realizadas con la misma escala. Pensamos que la especificidad del colectivo y de la materia puede explicar nuestros resultados.

Respecto al curso y los estudios previos, nuestros datos apuntan a la idea de que la actitud global hacia la estadística se empeora con los años de estudio de la misma, posiblemente porque encuentran dificultades con el tema.

En el caso de los estudios previos (4 niveles), el análisis factorial de varianza es significativo, las puntuaciones totales más bajas se presentan en las actitudes de los que nunca estudiaron estadística, confirmando nuestra teoría que es preciso enseñar este tema en el currículo de primaria y secundaria.

A la vista de las anteriores conclusiones, la información obtenida, a pesar del tamaño limitado de la muestra, nos permite realizar una primera aproximación a las actitudes hacia la Estadística en estudiantes de psicología.

En nuestro trabajo los resultados obtenidos cuanto menos nos deben incitar a la reflexión ya que son inferiores en todos los ítems a los obtenidos en otros trabajos similares en los que la muestra está formada por estudiantes de diferentes especialidades.

Esperamos, en consecuencia, que nuestros resultados animen a otros investigadores a proseguir analizando las dificultades de los estudiantes en la inferencia estadística y a proponer acciones educativas que contribuyan a mejorar el aprendizaje de estos conceptos.

\section{Agradecimientos}

Trabajo apoyado por el Proyecto EDU 2013-41141-P y financiado por Fundos Nacionais através da FCT - Fundação para a Ciência e a Tecnologia, no âmbito dos projetos UID/CED/00194/2013 e UID/MAT/00013/2013. También se agradece el apoyo del CIDTFF 
(LabDCT/UTAD) y Unidade de Investigação para o Desenvolvimento do Interior do Instituto Politécnico da Guarda (UDI/IPG).

\section{Referencias}

ANASTASIADOU, S. Affective reactions and attitudes of the last class of greek high school students towards statistics In: CERME, 4., 2005, San Feliu de Guicols. Proceedings of European Research in Mathematics Education. San Feliu de Guixols: M. Bosch (Ed.), 2005. p.1-10. Disponible en: $<$ https://pdfs.semanticscholar.org/7aa4/5eba1728b12dfd8dc024455d0051b66b2131 .pdf>. Acceso en: 1 mar. 2016.

APARICIO, A.; BAZÁN, J. Actitud y rendimiento en estadística en profesores peruanos. Acta Latinoamericana de Matemática Educativa, Sao Paulo, n. 19, p. 644-650, 2006 a.

APARICIO, A.; BAZÁN, J. L. Actitudes hacia la estadística en profesores de nivel primario. In: COLOQUIOS SOBRE MATEMÁTICA EDUCATIVA, 2006b, Lima. Actas... Lima: Pontificia Universidad Católica del Perú, 2006b. p. 127-133.

APARICIO, A.; BAZÁN, J. L. Aspectos afectivos intervinientes en el aprendizaje de la estadística: actitudes y sus formas de evaluación. Acta Latinoamericana de Matemática Educativa, Sao Paulo, n. 21, p. 180-189, 2008.

AUZMENDI, E. Las Actitudes Hacia la Matemática Estadística en las Enseñanzas Medias y Universitarias. 1 ed. Bilbao: Mensajero, 1992. 119 p.

BATANERO, C. Cap on va l'educació estadística? Biaix, Barcelona, n. 15, p. 2-13,1999.

CARMONA, J. Una revisión de las evidencias de fiabilidad y validez de los cuestionarios de actitudes y ansiedad hacia la estadística. Statistics Education Research Journal, v. 3, n. 1, p. 5-28, 2004.

CAZORLA, I. M. et al. Adaptaçao e validaçao de uma escala de attitudes em relaçao à estatística. In: CONFERÊNCIA INTERNACIONAL: EXPERIÊNCIAS E PERSPECTIVAS DO ENSINO DA ESTATÍSTICA, 1, 1999, Florianópolis. Procedings.... Florianópolis: Santa Catarina, 1999. p.45-57.

CUESTA, M.; RIFÁ, H.; HERRERO, F. J. Un estudio exploratorio, en estudiantes de psicología, de una escala de actitudes hacia la estadística. In: CONGRESO DE METODOLOGÍA DE LAS CIENCIAS SOCIALES Y DE LA SALUD, 7., Madrid, 2001. Póster presentado en el VII Congreso de Metodología de las Ciencias Sociales y de la Salud. Madrid, 2001.

ELMORE, P. B. ; VASU, E. S. Relationship between selection variables an statistics achievement. Journal of Educational Psychology, Washington, n. 72, p. 457-467, 1980.

ELMORE, P. B.; VASU, E. S. A model of statistics achievement using spatial ability feminist attitudes and mathematics. Related variables as prediction. Educational and Psychological Measurement, Santa Barbara, n. 46, p. 215-222, 1986.

ESTRADA, A. Análisis de las Actitudes y Conocimientos Estadísticos Elementales en la Formación del Profesorado. 2002. f. Tesis (doctorado en Didàctica de la Matemàica) - Universitat Autònoma de Barcelona, Bellaterra 2002.

ESTRADA, A. Actitudes hacia la estadística: un estudio con profesores de educación primaria en formación y en ejercicio. In: CAMACHO, M.; FLORES, P.; BOLEA, P. (Ed.). SEIEM, 11., 2007, San 
Cristóbal de la Laguna. Actas... Tenerife: Sociedad Española de Investigación en Educación Matemática, 2007. p. 121-140.

ESTRADA, A. Las Actitudes Hacia la Estadística en la Formación de los Profesores. 1. ed. Lleida: Milenio, 2009. 360 p.

ESTRADA, A.; BATANERO, C; FORTUNY, J. M. Un estudio comparado de las actitudes hacia la estadística en profesores en formación y en ejercicio. Enseñanza de las Ciencias, Barcelona, v. 22, n. 2, p. 263-274, 2004.

ESTRADA, A.; BATANERO, C. Explaining teachers' attitudes towards statistics. In: BATANERO, C. et al. (Ed.). Teaching Statistics in School Mathematics. Challenges for Teaching and Teacher Education. ICMI/IASE Study, 18., 2008, Monterrey. Proceedings of the ICMI Study 18 Conference and IASE 2008 Round Table Conference. Monterrey: International Commission on Mathematical Instruction e International Association for Statistical Education, 2008. p. 126-131.

ESTRADA, A.; BATANERO, C.; LANCASTER, S. Teachers' attitudes towards statistics. In C. BATANERO, C. et al. (Ed.). Teaching statistics in school mathematics. Challenges for teaching and teacher education. New York: Springer p. 173-174.

ESTRADA, A.; BAZÁN, J. L.; APARICIO, A. Un estudio comparado de las actitudes hacia la estadística en profesores españoles y peruanos. UNION, Igliori, n. 24, p.45-57, 2010. Disponible en: <http://www.fisem.org/paginas/union/info.php?id=96>. Acceso en: 25 feb. 2016.

ESTRADA, A.; BAZÁN, J.; APARICIO, A. Evaluación de las propiedades psicométricas de una escala de actitudes hacia la estadística en profesores. AIEM. Avances de Investigación en Educación Matemtica-2032, Madrid, v. 3, n. 2013, p. 5-23, 2012.

GAL, I. Adult's statistical literacy: meaning, components, responsibilities. International Statistical Review, La Haya, v. 70, n. 1, p. 1-25, 2002.

GAL, I.; GARFIELD J. B. Monitoring attitudes and beliefs in statistics education. In: GAL, I.; GARFIELD, J. B. (Ed.). The Assessment Challenge in Statistics Education. Voorburg: Ed. IOS, Press, 1997. p. 37-51.

GAL, I.; GINSBURG, L. The role of beliefs and attitudes in learning statistics: towards an assesment framework. Journal of Statistics Education, Ohio, v. 2, n. 2, p. 1-15, 1994. Disponible en: <http://www.amstat.org/publications/jse/v2n2/gal.html>. Acceso en: 23 feb. 2016.

GIL FLORES, J. Actitudes hacia la estadística. Incidencia de las variables sexo y formación previa. Revista Española de Pedagogía, Madrid, n. 214, p. 567-590, 1999.

GÓMEZ CHACÓN, I. M. Matemática Emocional: los afectos en el aprendizaje matemático. 1. ed. Madrid: Narcea, 2000. 280 p.

LANCASTER, S. A study of preservice teachers' attitudes toward their role as students of statistics and implications for future professional development in statistics. In: BATANERO, C. et al. (Ed.). Teaching Statistics in School Mathematics. Challenges for Teaching and Teacher Education. ICMI/IASE STUDY, 18., 2008, Monterrey. Proceedings of the ICMI Study 18 Conference and IASE 2008 Round Table Conference. Monterrey: International Commission on Mathematical Instruction e International Association for Statistical Education, 2008. p. 126-131.

MARTINS, J. A.; NASCIMENTO, M. M.; ESTRADA, A. Estudio preliminar de las actitudes de profesores portugueses hacia la Estadística. In: CONGRESO GALEGO DE ESTATÍSTICA E 
INVESTIGACIÓN DE OPERACIONS, 9., 2009, Ourense. Actas del IX Congreso Galego de Estatística e Investigación de Operacions, Ourense: COTO, R. T. et al. (Ed), 2009. p. 31-36.

NASCIMENTO, M. M.; MARTINS, J. A.; ESTRADA, A. Terra das maravilhas... As atitudes face à Estatística em estudantes universitários do norte de Portugal. In: COLÓQUIO INTERNACIONAL DE PSICOLOGIA E EDUCAÇÃO, 12., 2012, Lisboa. Actas do $\mathbf{1 2}^{\circ}$ Colóquio Internacional de Psicologia e Educação - Educação, aprendizagem e desenvolvimento: olhares contemporâneos através da investigação e da prática [CD-ROM]. Lisboa: ISPA - Instituto Universitário, 2012. p. 16781692.

MCLEOD, D. B. Affective issues in mathematical problem solving: some theoretical considerations. Journal for Research in Mathematics Education, Washington, DC, n. 19, p. 134-140, 1988.

MCLEOD, D. B. B. Attitudes and emotions: new view of affect in mathematics education. In: MC LEOD, D. B.; ADAMS, V. M. (Ed.). Affect and Mathematical Problem Solving: a New Perspective. New York: Ed. Springer-Verlag, 1989. p. 245-258.

MCLEOD, D. B. Research on affect in mathematics education: a reconceptualization. In: GROWS, D. A. (Ed.). Handbook of Research on Mathematics Teaching and Learning. New York: Ed. Macmillam N.C.T.M., 1992. p. 575-596.

MCLEOD, D. B. Research on affect and mathematics learning in JRME: 1970 to the present. Journal for Research in Mathematics Education, Washington, DC, v. 25, n. 6, p. 637-647, 1994.

MOORE, C. M. Group Techniques for Idea Building. 1 ed. Newbury Park: Sage, 1987. 136 p.

MORALES, P. Medición de Actitudes en Psicología y Educación. 1. ed. San Sebastián: Universidad de Comillas, 1988. 653 p.

NASSER, F. M. Structural model of the effects of cognitive and affective factors on the achievement of Arabic-speaking pre-service teachers in introductory statistics. Journal of Statistics Education, Ohio, v. 12, n. 1, p. 1-28, 2004. Disponible en: 〈www.amstat .org/publications/jse/>. Acceso en: 26 feb. 2016.

ONWUEGBUZIE, A. J. Teachers' attitudes toward statistics. Psychological Reports, New York, n. 83, p. 1008-1010, 1998.

ONWUEGBUZIE, A. J. Modeling statistics achievement among graduate students. Educational and Psychological Measurement, New York, v. 63, n. 6, p. 1020-1038, 2003.

OTTAVIANI, G. Promover la enseñanza de la estadística: La contribución del IASE y su cooperación con los países en vías de desarrollo. In: EXPERIÊNCIAS E PERSPECTIVAS DO ENSINO DA ESTATÍSTICA, 1999, Florianópolis. Actas de la Conferência Internacional: Experiências e Perspectivas do Ensino da Estatística. Florianópolis: Universidade Federal de Santa Catarina, 1999. p.1-7.

PHILIPP, R. A. Mathematics teachers' beliefs and affects. In: LESTER, F. (Ed.). Second Handbook of Research on Mathematics Teaching and Learning. Charlotte: Ed. Information Age Publishing y National Council of Teachers of Mathematics, 2007. p. 257-315.

ROBERTS, D. M.; BILDERBACK, E. W. Reliability and validity of statistics attitudes survey. Educational and Psychological Measurement, New York, n. 40, p. 235-238, 1980.

SCHAU, C. et al. The development and validation of the survey of attitudes towards statistics. Educational and Psychological Measurement, New York, v. 55, n. 5, p. 868-875, 1995. 
TANUR, J. M. La Estadística una guía de lo desconocido. 1. ed. Madrid: Alianza Editorial, 1992. $407 \mathrm{p}$.

VERA, O. Y.; DÍAZ, C. Dificultades de estudiantes de psicología en relación al contraste de hipótesis. In: JORNADAS VIRTUALES EN DIDÁCTICA DE LA ESTADÍSTICA, PROBABILIDAD Y COMBINATORIA, 1., 2013, Granada. Actas de las Jornadas Virtuales en Didáctica de la Estadística, Probabilidad y Combinatoria. Granada: Departamento de Didáctica de la Matemática de la Universidad de Granada, 2013. p. 197-203.

WATSON, J. M. Profiling teachers' competence and confidence to teach particular mathematics topics: The case of chance and data. Journal of Mathematics Teacher Education, New York, v. 4, n. 4, p. 305-337, 2001.

WILENSKY, U. Learning probability through building computational models. In: PME CONFERENCE, 19., 1995, Recife. Proceedings of the PME. Recife: Organising Committee, 1995. p. 152-159. No aparece en el cuerpo del texto

WILENSKY, U. What is normal anyway? Therapy for epistemological anxiety. Educational Studies in Mathematics, New York, n. 33, p. 171-202, 1997.

WISE, S. L. The development and validation of a scale measuring attitudes toward statistics. Educational and Psychological Measurement, New York, n. 45, p. 401-405, 1985. 